\title{
Tendencias historiográficas sobre el problema agrario en Colombia siglo XX
}

\author{
Olga Marina García Norato ${ }^{1}$
}

\begin{abstract}
Resumen
Este artículo tiene como objetivo principal analizar tres tendencias de interpretación del problema agrario en el país, a partir de la historiografía existente sobre este inacabado tema, (i) identificando a la gran concentración de la tierra en las diversas formas, producto de diferentes fenómenos económicos, sociales y políticos (ii) analizando la fragmentación de la propiedad de la tierra vinculada a productos comerciales como el café (iii) desde la perspectiva de factores externos, particularmente el control de cambios, como instrumento transferente de rentas que estimula la consolidación de la gran propiedad.
\end{abstract}

Palabras clave: Problema agrario, colonización de tierras, historiografía, conflicto 


\title{
Historiographic trends on the agrarian issue in Colombia during the 20th century
}

\begin{abstract}
This paper aims to analyze three trends to interpret the agrarian issue in Colombia, based on the existing historiography on this uncomplete subject, by i) identifying the huge concentration of land in its various forms, as a result of different economic, social and political phenomena; ii) analyzing the fragmentation of land ownership in relation to commercial produce such as coffee; iii) from the perspective of external factors, particularly currency control, as an instrument of income transfer promoting big estate consolidation.
\end{abstract}

Key words: Agrarian issue, land settlement, historiography, conflict.

Recibido: 16 de marzo 2012

Aceptado: 03 de julio 2012

\section{lntroducción}

En general, la historiografía económica sobre el problema agrario en Colombia se ha centrado en tres grandes tendencias de interpretación: la primera, que ha sido la preponderante, persiste en identificar a la gran concentración de la tierra en las diversas formas, como el hecho histórico desencadenante de los distintos fenómenos económicos, sociales y políticos que mantienen en la encrucijada a la nación colombiana. La segunda, menos difundida que la tendencia anterior, enfatiza en la fragmentación de la propiedad de la tierra vinculada a productos comerciales como el café, como la llave que abre el despegue económico de la moderna sociedad colombiana, en un doble sentido, y la tercera, de reciente desarrollo, tanto por tendencias institucionalistas como por visiones histórico monetaristas, se esfuerza en justificar el problema agrario desde la perspectiva de factores externos, particularmente el control de cambios, como instrumento transferente de rentas que estimula la consolidación de la gran propiedad. 


\section{Tendencia uno}

La preponderante, que persiste en identificar a la gran concentración de la tierra en las diversas formas, como el hecho histórico desencadenante de los distintos fenómenos económicos, sociales y políticos que mantienen en la encrucijada a la Nación colombiana. A propósito Reyes Posada explica el problema agrario en los siguientes términos: «a partir de la capacidad que crean las formas históricas de apropiación de la tierra y las modalidades de subordinación y resistencia del campesinado a tales procesos, para explicar la dinámica de los conflictos sociales» (Reyes Posada, 2009: 57).

Por su parte, Bejarano asume que el problema agrario y el malestar rural, deben buscarse en el interior de las haciendas, como una prolongación de la fragmentación de viejos latifundios de origen colonial o a partir de tierras baldías (Bejarano, 1987: 204).

Cabe destacar los datos referidos a la distribución del área nacional que hiciera hacia 1900 el geógrafo Vergara y Velasco los cuales registraron las siguientes cifras:

\begin{tabular}{|l|l|}
\hline $39.65 \%$ & $\begin{array}{l}\text { tierras inútiles por muchos } \\
\text { años }\end{array}$ \\
\hline $1.72 \%$ & $\begin{array}{l}\text { tierras de particulares, } \\
\text { inútiles }\end{array}$ \\
\hline $34.10 \%$ & de baldíos despoblados \\
\hline $5.70 \%$ & casi incultas \\
\hline $3.44 \%$ & apenas cultivadas \\
\hline $3.44 \%$ & bien cultivadas \\
\hline $2.58 \%$ & de pastos naturales \\
\hline $0.86 \%$ & de pastos artificiales \\
\hline
\end{tabular}

Estimaba igualmente que existían 120.000 fundos de menos de 10 hectáreas y 15.000 de más de 1.000 hectáreas, localizadas sobre todo en Cundinamarca y Boyacá" (Vergara, 1974: 801).

Señalaba la existencia de 3.000 hacendados y cerca de 180.000 estancieros dueños de pequeñas heredades, a lo que agregaba: «En Colombia domina el régimen de la gran propiedad, de manera que en las cercanías de la capital se enumeran fundos de millares de hectáreas... En algunos puntos los propietarios de cortas parcelas son numerosos, pero en torno de los principales centros poblados sucede lo contrario» (Vergara, 1974: 771).

Por otra parte, Otto Burguer sustenta una opinión contraria, y se refiere no solo al área de colonización cafetera; en la cual «las fincas pequeñas y medianas son la regla, mientras que la gran propiedad constituye la excepción» (Burguer, 
1971: 16); esto no deja de ser curioso. Los años transcurridos entre la opinión de Vergara y no de Burguer han presenciado parte de la colonización cafetera, ello no ha cambiado esencialmente la distribución de la propiedad, pero si la imagen que se tiene sobre ella.

Machado, va más allá: el problema no se resuelve con el cambio de la estructura de la propiedad rural como un acto del mercado o de intervención del Estado; o con la simple extinción del dominio de las propiedades adquiridas ilícitamente, o la expropiación con indemnización de aquellas que no cumplen una función social o que generan conflictos irresolubles entre los actores. «Además de reestructurar la propiedad por una decisión política, se requiere desmontar los factores que alimentan la concentración de la propiedad, la fragmentación antieconómica de ella, la exclusión de los que no tienen tierra para producir; los factores institucionales, políticos y privados que sostienen y estimulan una estructura agraria desigual, excluyente, antidemocrática, que se ha constituido en una afrenta contra la pobreza y el desarrollo» (Machado, 2005: 85).

Los historiales sobre la tenencia y el mercado de tierras en Colombia se pueden circunscribir a dos grandes aspectos: el intento de poner tributos progresivos a la propiedad rural, y la reforma agraria. Ambos procesos han fracasado en movilizar la tierra como factor productivo y en constituir un verdadero mercado de tierras con elementos económicos de formación de precios y dinamización de la oferta y la demanda. También fracasaron en crear elementos para corregir la desigualdad en la apropiación de la tierra reflejada en una estructura agraria bimodal (Johnston, 1980: 304).

En Colombia se han adelantado varias propuestas para gravar la propiedad rural como una elección para activar el mercado de tierras y presionar a los grandes propietarios a que las ofrezcan en el mercado. En 1949 una Misión del Banco Mundial encabezada por Lauchlin Currie (BIRF, 1950) hizo el primer contacto al tema sin que tuviera éxito su propuesta de aplicar tributos a la propiedad rural.

La idea de Currie no fue admitida por los terratenientes ni por los especialistas fiscales y administradores del país, por impracticable. Se continuó aplicando el gravamen existente del 4 por mil sobre los avalúos prediales, y el Comité de expertos que el gobierno designó para el estudio de la propuesta recomendó que se fortaleciera el Instituto Geográfico y se aceleraran los trabajos de inspección predial.

La frustración en la década del cincuenta para gravar la propiedad rural no consistió tanto en que las medidas se enderezaran contra la clase dirigente, «sino que la debilidad de la imposición sobre las tierras consiste en que, aunque despierta la oposición de los intereses agrarios, no ofrece un incentivo evidente para cualquier otro grupo social importante» (Hirschman, 1963: 963). 
A partir de los años sesenta Colombia ha estado utilizando la reforma agraria sin éxito notable y sin poder solucionar los problemas derivados de la tenencia de la tierra. La reforma agraria de los años sesenta, ley 135 de 1961, ley $1^{\text {a }}$ de 1968, fue distribucionista y marginal; sólo hizo una reparación superficial de la estructura agraria. La ley $4^{\mathrm{a}}$ de 1973 frenó los procesos de reforma agraria y la ley 35 de 1982 buscó agilizar los trámites para entrega de tierras en zonas de conflicto a través del Plan Nacional de Rehabilitación.

La ley 30 de 1988 introdujo cambios importantes en el proceso de reforma agraria, pero mantuvo el espíritu de la ley 135 de 1961, e intentó remover los obstáculos legales y de procedimiento que hicieron impracticables las leyes anteriores. Esta reforma siguió siendo marginal, pese a que trató de afectar directamente los últimos vestigios del latifundio tradicional, sin importar si las tierras estaban o no explotadas adecuadamente.

La nueva ley de reforma agraria, ley 160 de 1994, puede entenderse como la adecuación de la intervención del Estado para regular la estructura de la propiedad en un esquema de economía más abierta e internacionalizada. Se busca sustituir la injerencia directa del Estado por la evolución del mercado asistido de tierras, creando algunos alicientes a los agentes privados para que participen en él. La intromisión directa se deja para casos muy particulares de conflictos o de ubicación de desplazados por la violencia. La ley establece un subsidio a la compra de tierras por parte de campesinos y trabajadores sin tierra, y se abren líneas de crédito para compra de tierras para pequeños productores con tasas de interés cercanas a las del mercado. Esta alternativa de acceso a la propiedad también ha fracasado para resolver el problema de la tenencia de tierras.

Estos intentos de reforma agraria, que llevan 40 años, no han logrado una evolución significativa en la estructura de la propiedad. El ejercicio del mercado asistido de tierras está condicionado a la disponibilidad de recursos del Estado para otorgar los subsidios a la compra de tierras, por tanto su dinámica es lenta.

Paralelo a ello se ha producido una contrarreforma agraria por la compra masiva de tierras por parte de los narcotraficantes y la expulsión de cerca de más de un millón de personas del campo en los últimos 10 años.

Colombia ha pasado así a este nuevo siglo, sin resolver los problemas que le genera una estructura de la propiedad y la tenencia marcadamente desigual y bimodal, y un mercado de tierras caracterizado por distorsiones en la formación de precios, inflexibilidad en la oferta y presencia de factores extra-económicos en la posesión y uso de la propiedad.

Así las cosas, concurre una afirmación que ya hace parte de la historia: Colombia no ha resuelto su problema agrario. Este problema no es sólo el de 
la tenencia de la tierra, la cuestión agraria se ha hecho más compleja a medida que avanza la sociedad, los mercados, la tecnología y que la globalización se impone en todos los órdenes de la actividad humana. El problema agrario es la carencia de una decisión política de reformar la estructura agraria en función de objetivos de desarrollo y equidad de largo plazo. Es la no consideración del sector agropecuario como un sector estratégico para la sociedad, tanto en términos de seguridad alimentaria como de dominio territorial a través de actividades económicas lícitas, y también en calidad de sector que contribuye con externalidades significativas en la conservación del medio ambiente y calidad de vida.

La cuestión agraria no sólo debe mirarse más allá de la tenencia de la tierra, también implica situarla hoy en una concepción de lo rural más allá de lo sectorial como lo propone el paradigma de la nueva ruralidad que busca revalorizar la multifuncionalidad de la agricultura. Pero igualmente, implica situarse en el nuevo contexto del funcionamiento de un sistema agroindustrial que opera con unas relaciones de poder que están por fuera de la agricultura y donde el tema de la tierra como factor productivo desciende en la jerarquía de los elementos que configuran las relaciones de producción dominantes y el poder de decisiones en la estructura (Machado, 2002: 90).

La realidad es que el campo colombiano, escenario del conflicto armado, ha sufrido importantes trasformaciones en los últimos años. Hay una tendencia regresiva de los cultivos transitorios mientras que los de ciclo largo evidencian un fortalecimiento. Esta tendencia ha estado asociada a conflictos en torno a la tierra, al desplazamiento, a precarias relaciones laborales y, a los subsidios o apoyos estatales. Es el caso hoy tan en boga, de las plantaciones para la producción de agro combustibles a partir de la caña de azúcar y la palma aceitera (Mejía, 2008: 103).

En Colombia, campesinos, aparceros, indígenas, trabajadores agrícolas, negros, tienen una larga y dura historia de contienda y confrontación con terratenientes, empresarios agrícolas y empresas trasnacionales. Son muchos los hechos que recuerdan esta historia. Es conocida la Masacre de las Bananeras en 1928, durante el movimiento huelguístico que lideró el destacado dirigente agrario Raúl Mahecha, y que Gabriel García Márquez recuerda magistralmente en Cien Años de Soledad.

\section{Tendencia dos}

Menos difundida que la tendencia anterior, enfatiza en la fragmentación de la propiedad de la tierra vinculada a productos comerciales como el café, como la llave que abre el despegue económico de la moderna sociedad colombiana, en un 
doble sentido. Zuleta le otorga «significación histórica a la expansión cafetera, su trascendencia y originalidad consisten, en que con ella se da el fenómeno insólito de la combinación de la pequeña propiedad familiar y la producción para el mercado mundial. Hasta entonces todos los frutos de exportación, casi sin excepción, habían sido producidos en grandes propiedades, fuera por medio de esclavos, de siervos, de aparceros, agregados, arrendatarios, o por medio de peones asalariados...-entonces- salta a la vista el carácter cualitativamente nuevo de la coincidencia de pequeña propiedad y producción para la exportación que se da en el caso del café en el área de la colonización antioqueña... nos parece ser el factor decisivo y el más rico en proyecciones» (Zuleta, 1977: 163).

El proceso de parcelación iniciado durante la administración Olaya Herrera y que continuó después de expedida la Ley 200 tuvo un impacto reducido sobre la propiedad rural. Al respecto Antonio García establece: «Todas las parcelaciones que se realizaron en el país hasta 1940 favorecieron a poco más de 20.000 propietarios, distribuyendo unas 430.000 has. El número de propietarios favorecidos equivalía a sólo el 3.2\% de los dueños y al $6.5 \%$ de los arrendatarios y colonos registrados en el Censo de población de 1938 en el sector agropecuario. En Cundinamarca y Tolima el proceso de parcelaciones contribuyó decididamente, sin embargo, a fomentar la pequeña producción cafetera, aunque sin reducir el número de haciendas productoras del grano» (García, ).

Cabe resaltar en esta tendencia historiográfica del problema agrario en Colombia, uno de los planteamientos fundamentales de Marx, en el cual subraya la expropiación de los pequeños productores como fuente esencial de la acumulación. Bejarano menciona al respecto:

Tal como lo hemos visto en Colombia, durante las dos primeras décadas del siglo XX y por lo menos las tres últimas del siglo XIX hay un proceso de apropiación de la tierra, de formación de la gran propiedad territorial que no conlleva, con la misma intensidad, un proceso de expropiación en cuanto se apoya fundamentalmente sobre la expansión de la frontera agrícola y no sobre la expropiación de los pequeños productores, ello por lo demás responde a las particularidades del proceso de formación de la propiedad territorial en el país; desde luego no se niega la expropiación y la violencia a lo largo del siglo XIX; indicamos solo la divergencia en la intensidad de la apropiación y la expropiación toda vez que la primera se produce sobre tierras públicas o eclesiásticas no ocupadas en su totalidad. Más bien podría sostenerse la formación de una capa de pequeños productores paralela a la formación de la gran propiedad territorial desde la segunda mitad del siglo XIX (Bejarano, 1979: 21-22).

Lo específico del proceso colombiano es la apertura del mercado interior, no a partir de la conversión de los pequeños propietarios en asalariados sino a partir de la producción cafetera consolidada desde la pequeña propiedad. 
A mediados del siglo XX la economía colombiana crecía bien, impulsada por los buenos precios externos del café, fase que comenzó al final de la Segunda Guerra Mundial y persistió hasta 1955. «Era también una economía que estaba en una temprana fase de desarrollo y que avanzaba rápidamente en un intenso proceso de urbanización y migración, diferenciación campo ciudad e inversiones públicas para completar su infraestructura básica de vías, energía y aguas, todo lo cual aumentaba el comercio y las posibilidades de profundizar la especialización del trabajo en todos los resquicios antes aislados de la sociedad y con ello incrementaba la productividad de todos los factores» (Kalmanovitz y López, 2006: 84).

El desarrollo económico en Colombia se consolidó en los primeros años de la posguerra, la población dejó de ser predominantemente rural para concentrarse en las ciudades y la economía dejó de ser agrícola, para convertirse en urbana con cierto grado de complejidad en la división del trabajo y en la especialización." El crecimiento de las ciudades alcanzó su máxima velocidad en los cincuenta, impulsado por la migración de la población rural que, además del natural proceso de diferenciación entre campo y ciudad, y dentro de la misma agricultura, huía también de la violencia”.

«Entre 1917 y 1930 exceptuando 1922, los salarios experimentan un crecimiento sostenido que solo se interrumpirá ante el desempleo creado por la crisis». En 1928 anotaba el Ministro de Hacienda de Colombia que: «el salario mínimo de las obras públicas era de un peso diario, en tanto que había regiones del país donde el salario agrícola era de veinte centavos» (Urrutia, 1970: 34).

A propósito del salario agrícola, en éste período la Sociedad de Agricultores de Colombia (SAC), pide al gobierno: «nivelar por lo bajo los salarios de las obras públicas con los salarios de la agricultura» (El Tiempo, 1928).

\section{Tendencia 3}

Esta de reciente desarrollo, tanto por tendencias institucionalistas como por visiones histórico monetaristas, se esfuerza en justificar el problema agrario desde la perspectiva de factores externos, particularmente el control de cambios, como instrumento transferente de rentas que estimulan la consolidación de la gran propiedad. Así, Kalmanovitz y López, por ejemplo, aducen que una de las bases del modelo de desarrollo económico del siglo XX refiere: «el control de cambios fue otra herramienta fundamental que sirvió para imponerle a todas las empresas ellas mismas protegidas, que hicieran sus compras de materias primas a proveedores nacionales. También se gravaron muchos productos agrícolas con impuestos de importación con el fin de utilizar el gravamen en el fomento de la producción» (Kalmanovitz y López, 2006: 87). 
Los beneficiados por la protección eran escogidos por el gobierno de turno adjudicándoles un subsidio, contenido en el exceso del precio interno sobre el precio internacional del bien, arancel promedio del $40 \%$ pagado inadvertidamente por sus consumidores. La protección se convertía en un impuesto invisible que aumentaba la rentabilidad de las actividades que florecían bajo su manto, al tiempo que los ingresos del gobierno aumentaban sin tener que recurrir a la tributación transparente de los ciudadanos.

Las licencias de importación, obran como otro elemento de racionamiento que permitía a ciertas empresas e individuos capturar las divisas necesarias para ejecutar sus inversiones o adquirir sus bienes intermedios no producidos en el país, mientras que los perdedores debían resignarse a no poder contar con las importaciones que requerían. Las importaciones de bienes de consumo eran mínimas y su virtual prohibición extendió un medio propicio para que prosperara el contrabando, informalizando buena parte del comercio exterior del país.

En general, señala Kalmanovitz que «La experiencia del control de cambios y la expansión monetaria durante la gran depresión de los años treinta, (que en todas partes sembraron dudas sobre la capacidad de los mecanismos de mercado para propiciar el crecimiento), así como las intervenciones estatales impulsadas por las necesidades del país frente a las limitaciones a su comercio que le impuso la segunda guerra mundial, se combinaron para albergar un consenso político sobre la conveniencia de tener, instrumentos públicos de planificación racional de la actividad económica. Como ya se vio, la república conservadora combinó la alta protección a la agricultura y a la industria con un sistema de banca central de fomento por medio del crédito subsidiado y, finalmente, con la ampliación de las actividades del Instituto de Fomento Industrial IFI, la nacionalización de la Concesión de Mares, la fundación de ECOPETROL, y el establecimiento de la siderurgia de Paz del Río como un escalón en la conquista de la industria pesada» (Kalmanovitz y López, 2006: 87).

Teniendo en cuenta la referencia anterior, cabe resaltar la observación que hicieron el presidente de la Sociedad de Agricultores de Colombia Carlos Ossa Escobar y su secretario general y editor de la Revista Nacional de Agricultura, Gabriel Montes Llamas en los siguientes términos:

La Junta monetaria no se ha desvivido precisamente por financiar la producción o la comercialización de productos agropecuarios, todo lo contrario; después de realizar un asalto inmisericorde a los recursos del Fondo Financiero Agropecuario, erosionando su base de recursos para favorecer a industrias y entidades financieras en problemas, se rasga las vestiduras por la precaria situación del mismo. La respuesta clásica de ésta entidad, es incrementar las tasas de interés del Fondo Financiero 
Agropecuario; no como creen los agricultores por imposición del Fondo Monetario Internacional sino seguramente por influencia del Banco de la República, que después de haber realizado la operación de salvamento del sector financiero desea presentar una situación equilibrada en el FFAP a costa de los agricultores. La filosofía de la «eficiencia», de la ortodoxia, se aplica así al sector más competitivo de la economía, mientras que a los sectores protegidos se les socializan sus pérdidas a través de la emisión monetaria. Se consagra de nuevo la filosofía maniquea, en la cual la posesión de activos financieros o industriales es deseable, mientras que la posesión de la tierra y su posesión es retardataria e injusta (Ossa Escobar y Montes Llamas, 1985: 9).

Así las cosas, plantean para el sector agropecuario colombiano dentro de ésta tendencia, el problema visto desde la perspectiva de la no inclusión del sector como prioritario para la orientación política institucional, especialmente la política monetaria, que asumía la junta directiva del Banco de la República para controlar entre otros aspectos, las tasas de interés y tasas de cambio, que favorecieran la inversión de los sectores económicos más importantes del país.

Una segunda base del modelo de desarrollo que surge con la nueva república conservadora es el cambio de función del Banco de la República, la cual se define, a partir de 1951, como de fomento. El banco asignará crédito directamente al sector privado con subsidios en el costo de los recursos, utilizando cada vez más la emisión con tal propósito. El gobierno reducía los costos del crédito dirigido hacia algunos agentes, también escogidos por los funcionarios del banco central y bajo las directrices del gobierno, y con ello la tasa de interés se tornó también en una señal atrofiada, desincentivando el ahorro del público y haciendo más llano el sistema financiero.

Para ello se hicieron flexibles algunas de las facultades que el Banco tenía, y se le asignaron nuevas funciones sobre la fijación de los cupos de crédito, la determinación del descuento y de las tasas de interés, los porcentajes de encaje legal y las condiciones de elegibilidad de las obligaciones bancarias. Mediante estas atribuciones se consolidó su función como banco central, con amplias facultades para el diseño y ejecución de la política monetaria y crediticia del país.

Las pequeñas y medianas empresas encontraron el crédito racionado y caro porque los bancos escasamente recurrían a los depósitos del público, de tal modo que la oferta de recursos se estancaba mientras la demanda aumentaba a la par con el crecimiento económico del país. En últimas no se obtuvo lo que se había buscado en un comienzo, el resultado fue más bien una industrialización más dependiente de la política, que de la habilidad empresarial y que se vio obstruida para obtener aumentos de su productividad y para mantener los estándares internacionales de calidad. 


\section{Conclusiones}

En Colombia, por medio de disposiciones legales y métodos económicos, se pretendió lograr desde las primeras décadas del siglo XX, la distribución y titulación de uno de los elementos más importantes de la producción como es la tierra, sin resultados exitosos a pesar de la orientación política - institucional y legal que acomodaron los gobiernos de turno.

Durante el siglo pasado, el campesinado colombiano empeoró sus condiciones de vida y considerables extensiones de territorios e importantes ecosistemas han sido destruidos por los procesos de colonización que propiciaron las políticas agrarias.

El café acabó en Colombia con los salarios irrisorios, tenía razón Restrepo al indicar que la competencia elevaría los salarios. En efecto el periodo de expansión, tanto cafetera como de actividades urbanas evidencia, en conjunto, una ostensible alza de salarios.

Desde finales de los años 40 se venían adoptando decisiones encaminadas a ampliar las funciones del Banco en materia de regulación y ejecución de la política monetaria y crediticia. Tales medidas se concretaron mediante el Decreto legislativo 756 de 1951, el cual le otorgó al Banco atribuciones para diseñar políticas monetaria, crediticia y cambiaria, a fin de crear condiciones propicias para el desarrollo ordenado de la economía colombiana.

\section{Referencias Bibliográficas}

Bejarano, Jesús Antonio. 1987. Ensayos de historia agraria colombiana. Bogotá: CEREC.

Bejarano, Jesús Antonio. 1984. La Tierra y el poder político: la reforma agraria y la reforma rural. Bogotá: FAO.

Bejarano,, Jesús Antonio. 1979. El régimen agrario de la economía exportadora a la economía industrial. Bogotá: Ed. La Carreta.

Currie, Lauchlin, Misión del BIRF. El Tiempo, mayo 12 de 1928

Fajardo Montaña, Darío. 1984. Tierra, poder político y reformas agraria y rural. la reforma agraria y la Reforma Rural. FAO.

Fals Borda, Orlando. 1975. Historia de la cuestión agraria en Colombia. Bogotá: Publicaciones de la Rosca. 


\section{Olga Marina García Norato}

Tendencias historiográficas sobre el problema agrario en Colombia siglo xx, artículo xxxxx

Hirschman, Albert. 1967. «El impuesto sobre tierras y reforma agraria en Colombia». En Richard M. Bird y Oliver Oldman (comps.). La imposición fiscal en los países en desarrollo. México: Uteha..

Johnston, Bruce y Peter Kilby. 1980. Agricultura y transformación estructural. México: Fondo de Cultura Económica.

Kalmanovitz, Salomón y Enrique López. 2006. La Agricultura Colombiana en el siglo $X X$. Bogotá: Fondo de Cultura Económica.

Machado, Absalón. 2009. Ensayos para la Historia de la Política de Tierras en Colombia. De la colonia a la creación del Frente Nacional. Bogotá: Editorial Gente Nueva.

Machado, Absalón. 2005. "Apreciaciones no ortodoxas sobre la reforma agraria», Economía Colombiana, 309: 66-74. Ocampo, José Antonio (Comp.). 1996. Historia económica de Colombia. Bogotá: Siglo XXI. Ossa Escobar, Carlos y Gabriel Montes Llamas. 1985. El pensamiento económico y social de la SAC. Bogotá, Sociedad de Agricultores de Colombia.

Reyes Posada, Alejandro. 2009. La violencia y el problema agrario en Colombia. Bogotá: Editorial Norma.

Reyes Posada, Alejandro. 2009. Guerreros y campesinos, El despojo de la tierra en Colombia. Bogotá: Editorial Norma.

Urrutia, Miguel y Mario Arrubla. 1970. Compendio de estadísticas históricas de Colombia. Bogotá: Universidad Nacional de Colombia.

Zuleta, Estanislao. 1977. Conferencias sobre Historia económica de Colombia. Bogotá: Ed., La Carreta,. 